\title{
MedienPädagogik
}

Zeitschrift für Theorie und Praxis der Medienbildung

Themenheft Nr. 32: Offenheit in Lehre und Forschung - Königsweg oder Sackgasse? Herausgegeben von Franziska Bellinger und Anna Heudorfer

\section{Eine Frage der Qualität \\ Qualitätsforderungen an Open Educational Resources in Schule und Hochschule}

Jane Brückner

\begin{abstract}
Zusammenfassung
Die Nutzung und Bereitstellung von Open Educational Resources (OER) sind mit vergleichsweise hohen Unsicherheiten und Herausforderungen verbunden, die in der deutschen Bildungslandschaft zu einem erhöhten Bedarf an Qualitätsausweis für freie Bildungsmaterialien führen. Die Debatte um diese vermeintlich geringer-wertigen Unterrichtsmaterialien reisst nicht ab und qualitätssuggerierende Entwicklungen begleiten die Nutzung von OER in der Schulpraxis: Verlage und Hochschulen geben cc-lizenziertes Material an die Lehrkräfte in allen Bildungsbereichen, Plattformen für OER versuchen Qualitätsnachweise über Nutzerbewertungen, Bildungsinstitutionen entwerfen Qualitätskriterienmodelle für OER. Bei allen Unternehmungen dem Bedarf an Qualitätssicherung und Qualitätsausweisen nachzukommen, wird vernachlässigt, dass gerade OER und die konsequente Praxis von Open Education die Nutzer als autonome, kreative und kompetente Lehrenden und Lernenden versteht. Vor diesem Hintergrund steht ein kritisches Hinterfragen der aktuellen Implikationen des verwendeten Qualitätsbegriffes aus, sowie des Beteiligtenkreises für den Aushandlungsprozess von OER-Qualität.
\end{abstract}

A question of quality - Demands of quality for Open Educational Resources

\begin{abstract}
The use and provision of Open Educational Resources (OER) are associated with comparatively high levels of uncertainty and challenges, which are related with an increased need of quality certificates for OER in the German education landscape. The debate about these supposedly less valuable teaching materials does not stop and the use of OER in school practice is attended by quality suggesting developments: Publishers and universities provide cc-licensed material to teachers in all educational settings, platforms for OER try to give quality certifications via user ratings, educational institutions design quality models for OER. All these enterprises of satisfaction the need in qualityassurance and certification are neglect that OER and the consequent practice of Open Education the users understand as autonomous, creative and competent teachers and learners. Against this background, there is still to come a critical questioning of the current implications of the quality concept, which is used, as well as the participants of determination of quality.
\end{abstract}




\section{Qualität und Qualitätskriterien für OER}

Die deutsche Qualitätsdebatte um Open Educational Resources scheint nicht abzuflauen, auch wenn die Qualitätssicherung nicht mehr als die grösste Herausforderung für die Nutzung und das Erstellen von OER wahrgenommen wird. Vielmehr sind es nun kulturelle, rechtliche und technische Schwierigkeiten, die der Verbreitung von OER in der deutschen Bildungslandschaft entgegenstehen. Trotzdem begegnen Lehrende und Lernende (fremdem) Material mit zurückhaltender Skepsis. Insbesondere wenn das Material didaktisch aufbereitet wurde, wird von Seiten der Lehrenden an dessen Qualität gezweifelt und auf Angebote von Verlagen oder etablierten Bildungsinstitutionen wie Hochschulen zurückgegriffen. Hier greift bei den Nutzerinnen und Nutzern häufig ein individuelles Markenvertrauen in die Organisationen und Verlage als Qualitätsgarantie. Markenvertrauen setzt eine Beziehung zwischen einem Vertrauensgeber und einem Vertrauensnehmer voraus. Es ist bestimmt von stereotypen Erwartungen (z.B. Qualitätserwartungen) an das Verhalten des Vertrauensnehmers und überträgt diese auf die Produkte, insbesondere dann, wenn die Marktlage unübersichtlich und intransparent ist. Damit reduziert Markenvertrauen die Komplexität der Produktlage und bietet dem Nutzer Orientierung für seine Auswahlhandlungen. Die Anforderungen an OER sind zudem divers, denn Lernende wie Lehrende nutzen freie Bildungsmaterialien entlang ihrer institutionellen und individuellen Voraussetzungen. Somit ist die Qualitätsdebatte um OER mit einem mehrdimensionalen Netz an Akteuren, Perspektiven und Forderungen beschäftigt, das sich an den Bildungsinstitutionen (z.B.: Schule, Hochschule, Weiterbildungs-institutionen), an der Position und Rolle innerhalb der Bildungsorganisation (z.B.: Lernende, Lehrende und Entwickler), aber auch an etablierten Handlungsweisen (z.B.: Kooperation, Kollaboration und Teilen) und einer gewachsenen Lern- und Lehrkultur ausrichtet.

Lösungsansätze für das Vertrauensdefizit gegenüber den organisatorisch unabhängigen OER gibt es bereits. So sollen den Vorbehalten gegenüber der Qualität von nicht-institutionellen OER beispielsweise Nutzerbewertungen und strukturierendes Tagging auf den Webplattformen entgegenwirken ${ }^{1}$. Ein weiteres Beispiel für Qualitätssicherungsversuche sind die Zertifizierungen durch Experten und Expertengremien. Dieses Peer-Review-Verfahren ist von wissenschaftlichen Publikationen bekannt und wird nun auch durch Hochschulen und andere Bildungseinrichtungen auf freie Bildungsmaterialien übertragen, um das Vertrauen in die Qualität von OER durch das organisationsinhärente Qualitätsversprechen zu generieren. Selten wird dabei beachtet, dass diese Zertifizierung mit einem strategischen Mehrwert für die Bildungsinstitutionen einhergeht, die die eigenen Reputationen über OER systematisch abbildet und so den Marktwert der eigenen Bildungsinstitution in der Zielgruppenwahrnehmung erhöht. Entsprechend hoch ist die Motivation für das Erstellen von OER durch bildungsnahe Organisationen und Institutionen. OER geraten so jedoch leicht

1 Vgl. edutags.de. 
zum wirksamen Kommunikationsinstrument des Marketings und entfernen sich dadurch von einer Kultur des Teilens, «in der Medien ihren Status als feste Entitäten verlieren» (Unger 2012, 138). Grundsätzlich fliessen bei der institutionellen und organisatorischen Qualitätssicherung «[s]oziale Kontrolle und Instrumente der Vertrauensbildung durch Bewertung [...] ineinander» (Mau 2017, 150). Den kollaborativen Gedanken von OER jedoch befördern sie wohl eher nicht, da das Material über das Label der Bildungsinstitution an die Organisation und deren Qualitätsversprechen gebunden wird. Ein kreativer Umgang mit den Inhalten im Sinne des retain, reuse, revise, remix, redistribute (Wiley 2013) von OER wird erschwert, obwohl er nicht ausgeschlossen ist. Solche Mechanismen der Qualitätssicherung führen zu einem Verständnis von OER, das verstärkt die Distribution von Material und Inhalten forciert. Der Modifikations- und Anpassungsgedanke gerät in den Hintergrund.

Soll die Qualität vom Open Educational Ressource bestimmt oder gar gemessen werden, orientiert sich die Argumentation für ein Messinstrument häufig an einem produktorientierten Verständnis von Qualität: Diese Modelle zur Qualitätsmessung zeichnen sich durch eine skalare Struktur aus, anhand derer die Ausprägungen in den zu definierenden Merkmalen des jeweiligen Produktes zwischen gut und schlecht gemessen werden sollen. Demnach bestimmt sich die Qualität von OER vor dem Hintergrund eines produktorientierten Qualitätsverständnisses über dessen Eigenschaften und Bestandteile auf der Merkmalsebene (Knispel 2008, 18). Zentrale Fragen der Evaluation auf der Merkmalsebene widmen sich der Vollständigkeit, der Korrektheit und der Aktualität der Informationen, dem didaktischen Design und dem Angebot von Unterstützung, Hilfe und Feedback (Mau 2017, 31). Diese Betrachtung von Qualität bewegt sich auf der Evaluationsebene und hat den Fokus ihrer Sicht auf der Effizienz und der Kosten-Nutzen-Relation. Evaluationen haben die Funktionen zu kontrollieren, zu legitimieren und zu überprüfen, um darauf aufbauend optimierend in Entwicklungsprozesse einzugreifen (vgl. Stamm 2003, 55).

\section{Auf dem Weg zu einem Qualitätssicherungsmodell für OER}

Einen entsprechenden Versuch die Qualität von OER mit einem deutschen Modell messbar zu machen, unternimmt die Internationale Bestandsaufnahme von Instrumenten zur Qualitätssicherung von Open Educational Resources der Hamburger Open Online University. Die Studie vergleicht sechs Evaluationsmodelle und extrahiert aus diesen Qualitätskriterien, die «OER nicht wie herkömmliche Bildungsmedien behandeln» (Zawacki-Richter und Mayrberger 2017, 7), sondern (auch) die Spezifika von freien Bildungsmaterialien abzubilden im Stande sind. Dabei wird ein eingeschränktes Verständnis von OER zu Grunde gelegt: Die Überlegungen und Argumentationen gründen sich auf Lernmaterialien, die Teil von didaktischen Szenarien sind oder sein können (Zawacki-Richter und Mayrberger 2017, 15) und möglichst echte OER 
darstellen. Mit Verweis auf das Creative Commons Lizenzierungssystem und ein allgemeines Urheberrecht (Zawacki-Richter und Mayrberger 2017, 14) bleibt es jedoch im eigens für diese Studie publizierten Sonderband des Magazins Synergien bei einer überblickshaften Schau der Definitionen von OER. Das Objekt dessen, das eine Qualitätssicherung bedarf, bleibt auch mit den Ausführungen zum Verständnis von OER und Offenheit an der HOOU ungenau umrissen. Als Grundlage für ein deutsches Qualitätsmodell für OER - so immerhin der Anspruch der Autoren - kann das Verständnis von OER einer einzelnen Organisation nicht auf die deutsche Bildungslandschaft übertragen werden. Deshalb kann auch der Vorschlag, «ein deutsches Instrument zur Qualitätssicherung von OER» (Zawacki-Richter und Mayrberger 2017, 43) zu entwickeln, welches auf dem Kriterienmodell der HOOU aufbaut, nur zu einem Qualitätssicherungsinstrument führen, das Aussagen für solche OER liefert, die den offenen Bildungsmaterial der HOOU entsprechen. Für ein weiterführendes Instrument zur Qualitätssicherung müssen OER als Gegenstand der Qualitätssicherung umfassender definiert werden. Es bedarf für ein nationales Modell der Qualitätssicherung einer differenzierten Merkmalanalyse von OER, die das ganze Spektrum von Offenheit und Bildungsmaterialien abbilden. So wäre es denkbar, mit den in der Studie erwähnten 5 r's (retain, reuse, revise, remix, redistribute) zu arbeiten oder das Creative Commons Lizenz Spektrum ${ }^{2}$ für die Entwicklung eines OER-Qualitätsmodells zu nutzen, welches eine graduelle Offenheit von OER beschreibt und eine Dichotomie von echt/ unecht vermeiden. Darüber hinaus wären auch die Anforderungen von Schülerinnen und Schülern und Lehrkräften mit denen der Hochschulehrenden und Studierenden in Relation zu setzen. So wären zum Beispiel Aspekte der Berufsbefähigung und digital literacy im Hochschul- und Weiterbildungsbereich für eine Qualitätserfassung von OER sicher relevanter als in der (grund-)schulischen Bildung. Dagegen ist die an den Entwicklungsstufen angepasste didaktische Aufbereitung von freien Bildungsmaterialien ein weitaus grösseres Thema für die (grund-)schulische Bildung.

Mit dem Sonderband zur Qualität von OER wurde von der HOOU eine organisationsinterne Qualitätssicherung von OER durch die Autoren «als strategisch relevant für das Verbundprojekt identifiziert» (Zawacki-Richter und Mayrberger 2017, 7). Dies ist mit der vergleichenden Methode nachvollziehbar und sicher für die HOOU gewinnbringend. Das vorgestellte Kriterienmodell kommt dem organisationsinternen Bedarf einer Qualitätsstrategie entgegen und kann mit der Strukturierung nach Zielgruppenorientierung, Lernziele, Engagement und Motivation, Kooperation, Anwendung und Transfer, Medien- und Präsentationsvielfalt, Supportleistungen und Orientierung an Bildungsstandards auch in anderen Bildungsorganisationen für die Bewertung von OER genutzt werden.

Das Anliegen der Autoren ein deutsches Modell für die Qualitätssicherung von OER auf der Grundlage der vorgestellten Studie zu entwerfen, lässt die Frage

2 Abrufbar unter: https://commons.wikimedia.org/wiki/File:Creative_Commons_Lizenzspektrum_DE.svg. 
aufkommen, warum es gerade im deutschsprachigen Diskurs zu OER eine rege Diskussion um die Qualität freier Bildungsmaterialien gibt. Neben den Erklärungsversuchen im Zusammenhang mit der Rezeption der PISA-Studie, ist ein Blick in die OER-Praxis für die Beantwortung weiterführend. Dem Wunsch der Sicherung von Qualität freier Bildungsmaterialien kommen Normierungs- und Kontrollverfahren von vertrauensgenerierenden Institutionen, wie Verlagsmarken oder Bildungsinstitutionen zunehmend nach: Verlage geben zunehmend cc-Lizenziertes Material mit ihrem Label für den Unterricht heraus, Hochschulen bieten neuerdings OER für erweiterte Zielgruppen an. Beide Entwicklungen denken die Bereitstellung von OER als Dienstleistung und sind entsprechend mit einem ökonomischen Zweck verbunden: Mit Hilfe von OER sollen Bildungskunden gewonnen und gebunden werden. Dabei richten sich diese Dienstleister mit ihrem freien Bildungsmaterial sowohl an die Lehrenden und Lernenden von Hochschulen und Weiterbildungsinstitutionen als auch an die Schülerinnern und Schüler und Lehrkräfte der Schulen.

\section{OER in der Schulpraxis}

Nun handelt es sich bei OER jedoch um Bildungsmaterialien, die unabhängig von Bildungsorganisationen auch ein informelles, mithin individuelles Lernen ermöglichen sollen. Die Antwortversuche auf eine Frage nach der Qualitätssicherung von OER muss mit dieser Implikation von OER den Blick von den etablierten Qualitätssicherungspraxen der Verlage und Bildungsinstitutionen wegführen, hin zu den Akteuren und dem Material selbst, zur Open Educational Practice und zurück zu Open Educational Resources.

Tatsächlich sind es schulische Lehrkräfte, die das Teilen und Verbreiten von Übungsblättern und Ähnlichem nicht erst mit dem Aufkommen der CC-Lizenzen umsetzen. Mit Verweis auf die Granularität von OER ist festzustellen, dass die meisten im Netz zu findenden Materialien «sich auf einzelne Lehr-Lernaktivitäten [beziehen]: Das Übungsblatt zum Bruchrechnen, für die Grammatikregeln oder die Geschichtsdaten - angefertigt und digital bereitgestellt von einer Lehrkraft - gehören hierzu» (Kerres 2016, 2). Es handelt sich um «user-generated» Material, welches im Verhältnis zu «oragnisationally-produced resources» (Camilleri, Ehlers, und Pawlowski 2014, 10) eher von geringerem Umfang ist und mit weniger finanziellen und zeitlichem Aufwand erstellt wurde.

Gerade in der Unterrichtspraxis stehen diese OER in einem Qualitätsvergleich zum traditionellen Schulbuch und anderen Materialien von etablierten Bildungsorganisationen. Ihren Einsatz finden Materialien, die von Lehrkräften erstellt wurden, als Ergänzung in Exkursen oder Vertiefungen zu traditionellen Materialien. Sie sind in der Sichtweise der Qualitätssicherung ‘Kannmaterial`. OER im Umfang eines Arbeitsblattes müssen sich in diesem Zusammenhang in die curricularen Vorgaben der 
Länder und Schulen didaktisch einpassen. Eine solche Einpassung, Anpassung und auch Modifikation wird jedoch von den Lehrkräften selbst übernommen und ebenfalls verantwortet werden müssen. Dabei werden das Schulbuch erweiternde Materialien problemorientiert von den Lehrkräften recherchiert. Es sind mithin konkrete Fragestellungen, die sich aus dem Unterrichtsgeschehen ergeben und mit verfügbarem Material bedient werden. Im Zusammenhang mit der Heterogenität und der individuellen Lernförderung greifen Lehrende vermehrt auf online verfügbares Material zurück, um das didaktische Spektrum ihres Unterrichts zu erweitern und zu differenzieren. Sind diese Lehrkräfte für das deutsche Urheberrechtsgesetzt sensibilisiert, suchen und nutzen sie vermehrt cc-lizensiertes Material, um ihr berufliches Handeln innerhalb eines rechtssicheren Rahmens zu vollziehen. In diesen Zusammenhang wird eine Anspruchssteigerung an die Berufsprofessionalisierung wirksam, mit der Lehrende sich seit einigen Jahren konfrontiert sehen: Das Aufgabenspektrum erfährt ständig Erweiterung, die berufliche Rolle wird fortwährend modifiziert und das Eingebundensein der Lehrkräfte in das Bildungssystem muss sich immer wieder an neue Herausforderungen und gesellschaftlichen Wandlungsprozesse anpassen. ${ }^{3}$ Nicht mehr nur das Vorbereiten, Inszenieren, Durchführen und Auswerten von Unterricht, sondern auch das Beraten, Moderieren, Schlichten und Betreuen ausserhalb des Unterrichts, in der Elternarbeit, bei Projektarbeiten und kollegialen Interaktionen sind Teile des Aufgaben- und Handlungsfeldes von Lehrkräften. Dazu gehört spätestens seit 2000 auch die Beteiligung an der Schulentwicklung (KMK 2004).

Die Verwendung und Erstellung von OER tangieren Aspekte der professionellen Kompetenz von Lehrenden, indem sie Überzeugungen und Werthaltungen ansprechen und sogar in Frage stellen können. OER und ihre konsequente Nutzung entlang der 5 r's haben zudem das Potential das Professionswissen der Lehrenden zu erweitert und zu verändert, welches sich in den Bereichen Fachwissen, Fachdidaktisches Wissen, Pädagogisch-psychologisches Wissen, Organisationswissen und Beratungswissen ausgestaltet. Wie OER von Lehrkräften eingesetzt wird ist mit Blick auf die professionelle Kompetenzentwicklung eine für die Qualitätsdiskussion um OER weitreichende Frage, denn sie greift nicht nur auf den professionellen Kompetenzerwerb von Lehrkräften zurück, sondern öffnet die Debatte auch für die tatsächliche Berufsund Schulpraxis.

Hierzu ist festzustellen, dass das cc-lizenzierte Arbeitsblatt oder das dem Themenschwerpunkt entsprechende Bild- und Textmaterial als OER mit Blick auf die Effizienz innerhalb der eigenen Unterrichtsvorbereitung zwar recherchiert und für die Unterrichtspraxis genutzt wird, das Teilen und Onlinestellen des eigenen Materials aber weitaus weniger von den Lehrkräften umgesetzt wird. Diese Zurückhaltung kann mit einer Furcht vor der anonymen Bewertung durch Kollegen, Eltern, Ausbildern und Ministerien erklärt werden. Sie kann aber auch mit dem eigenen

3 Siehe zum Beispiel: Bildung in der digitalen Welt. Strategie der Kultusministerkonferenz 2016. 
Qualitätsanspruch der Lehrkräfte (Deimann und Bastiaens 2010) an das Material verstanden werden, welches das erstellte Unterrichtsmaterial nicht immer erfüllt, weil es individuell zugeschnitten oder unfertig ist. Im eigenen didaktischen Handeln können diese Unvollständigkeiten und vermeintlichen Qualitätsmängel in der Unterrichtspraxis von den Lehrenden ausgeglichen und angepasst werden: Das Material selbst gibt diese professionsbezogene Handlungskompetenz jedoch nicht wieder. Das Heraustreten in die digitale Öffentlichkeit ist jedoch mit einer Offenlegung und Transparenz verbunden, die das eigene Material in einen Bewertungsdiskurs geraten lässt, dem der Urheber des Materials durch die inhärente Verbundenheit von Content und Ersteller ebenfalls ausgeliefert ist, ähnlich wie beim Qualitätsvertrauen in die Bildungsinstitution. Zudem sind die Wahl der Lizenz und das Ausweisen dieser mit einer Komplexitätssteigerung der Weitergabe des eigenen Materials verbunden. Deshalb ist es nachvollziehbar, dass die Motivation des Teilens von Unterrichtsmaterialien innerhalb der jeweiligen Fachgemeinschaften zwar grösser als fachübergreifend ist. Aber auch hier bleibt der Austausch häufig auf den schulinternen und kollegialen Akteurskreis beschränkt (Deimann und Bastiaens 2010).

Nicht also nur das Unterrichtsmaterial ist der bewertenden Beobachtung der anonymen Rezipientenschaft ausgesetzt, sondern auch dessen Ersteller sieht sich der potentiellen Vielzahl der Rezipienten und Nutzer einer Qualitätsprüfung ausgesetzt, die unmittelbar mit der eigenen beruflichen Professionalität verbunden wird. Sowohl die Anzahl als auch das Spektrum der potentiellen Prüfer ist dabei gross: Lernenden und Lehrenden, Vorgesetzten, Fachexperten sowie Eltern werden vermeintlich zu potentiellen Bewertenden der didaktischen Leistungsfähigkeit. Damit sieht sich die Lehrkraft mit ihrem Material einer Vielzahl von bewertenden Perspektiven und Qualitätsansprüchen ausgesetzt. Dass in diese Ansprüche Wertmassstäbe einfliessen, die insbesondere bei Bewertenden innerhalb des Bildungssystems einem institutionellen Normierungsrahmen folgen, reduziert den Freiheitsgrad der Lehrkräfte im Zusammenhang mit der Freigabe von Unterrichtsmaterial zusätzlich. Systemisch gedacht, handelt es sich hierbei also nicht nur um eine Rollenverkehrung der Lehrenden vom Prüfer und Bewertenden hin zum Geprüften und Bewerteten, sondern eben auch um eine Potenzierung der Bewertungsansprüche, welche Lehrkräfte vor dem Zugänglichmachen des selbsterstellten Materials für alle zurückschrecken lässt. Um das eigene didaktische Handeln zu rechtfertigen und zu sichern, soll das Material nicht nur einen rechtssicheren Umgang ermöglichen, sondern eben auch Repräsentant für die Qualität des eigenen didaktischen Handelns sein. 


\section{Qualität und Qualitätssicherung von OER}

Unter dem Aspekt der Qualitätssicherung sind es eben diese (potentiellen) Ansprüche an das Unterrichtsmaterial, die Lehrkräfte auch bei der Nutzung von OER ihrer Auswahl für den eigenen Unterricht zu Grunde legen. Die Qualität für Bildungsmaterial wird als unverzichtbar für den Ausweis der eigenen beruflichen Praxis wahrgenommen. Deshalb greifen Lehrende gern auf (digitale) Lehr- und Lernmaterialien zurück, deren Einsatz im Unterricht anerkannt und von der Schulleitung und Ministerien empfohlen wird (vgl. Muuß-Mehrholz und Schaumburg 2014, 31). So können qualitative Unbestimmtheiten und Unsicherheiten umgangen werden, denn das Unterrichtshandeln gilt im Rahmen der Normen und Konventionen vor den Kontrollinstanzen Eltern, Schulleitung und Ministerien als gerechtfertigt.

Institutionelle Bildungsmarken wie Hochschulen und Verlage, Fachexperten mit akademischen Titeln haben diesbezüglich einen Vertrauensvorsprung gegenüber freien Materialien unter cc-Lizenz. Der Einsatz solcher gelabelten Materialien im Unterricht wird weniger kritisch hinterfragt und dient den Lehrenden der Vergegenwärtigung und Kommunikation der eigenen beruflichen Qualität.

Trotzdem nutzen Lehrkräfte zunehmend auch Material, welches cc-Lizenziert ist. Dabei sind sie «zunächst vor allem an dem kostenfreien Zugang und der rechtssicheren Verwendung von Lernmaterialien interessiert» (Kerres 2016, 1f.). Die Studie von Neumann zeigt zudem, dass auch der barrierefreie Zugang zum Material eine wesentliche Rolle für die Motivation der Nutzung von kostenlosem Material aus dem Internet bei Lehrkräften eine grosse Rolle spielt (Neumann 2015, 101). Diese kann von Verlagen und Universitäten ebenso erstellt sein, wie von Kollegen. Deshalb kommen etablierte Bildungsorganisationen und-institutionen dem Bedarf der Lehrkräfte zunehmend nach, indem sie Unterrichtsmaterial erstellen, die diese drei Kriterien erfüllen: Kostenfreiheit, Rechtssicherheit durch cc-Lizenzen und Qualitätsversprechen über die (auch personelle) Expertise etablierter Organisationen oder Unternehmen. Diese Materialien sind meist didaktisch aufbereitet und bieten den Lehrkräften den unmittelbaren Einsatz in der Unterrichtspraxis an. Dabei darf aber nicht übersehen werden, dass diese Zweckdienlichkeit an einer normativen Qualitätszuschreibung ausgerichtet ist. Materialien unter cc-Lizenz bedürfen einer eigenen, von ihrer didaktischen Zweckdienlichkeit unabhängigen Bewertung (vgl. Heid 2000). Diese Bewertung kann bei kollaborativ erstelltem und ständig modifizierbarem Material ebenfalls nur kollaborativ erfolgen; Qualität von OER muss kontinuierlich weiterverhandelt werden. Insbesondere bei OER ist Qualität einem fortdauernden Aushandlungsprozess unterworfen, der von den beteiligten Interessengruppen nicht festgeschrieben wird, sondern zu einem zwischenzeitlichen Konsens gerät. Diesem Konsens folgen weitere zwischenzeitliche Übereinkünfte von Qualitätsanforderungen, die von anderen Nutzern und Anspruchsgruppen erweitert und modifiziert werden. Eine Normierung der Qualität von OER widerspricht dieser ständigen Anpassungsbewegung der 
Qualität an die Partikularinteressen der beteiligten Akteure von OER im Prozess des Teilens und Veränderns. Sie widerspricht auch der sogenannten Granularität auf der Contentebene (vgl. oben). Es gilt also festzuhalten, dass ein Modell der Qualitätssicherung mit normativem Anspruch dem Material selbst nicht nachhaltig gerecht werden kann, da es in seiner inhärenten Statik dem fluiden Merkmal von OER widerspricht. Qualitätsnormen und -standards beschneiden die Autonomie des Nutzungsprozesses von OER, will Qualitätssicherung doch auch kontrollieren (Controlling) und steuern. OER und die implizite Open Educational Practice dagegen verweigern sich schon in ihrem zentralen Merkmal der Offenheit einer Steuerung, deshalb sollte auch die Qualitätsbestimmung im Aktionsfeld des Teilens generiert werden. Nur dann wäre der Autonomie von OER und ihrer konsequent gelebten Praxis entsprochen.

Mehr noch: Gerade Lehrenden darf man zutrauen, dass sie ihre didaktischen und pädagogischen Kompetenzen nutzen, um gutes Material für ihre Lernenden angemessen zu kreieren, zusammenstellen und weiterzuentwickeln. Gerade Ihnen ist es bewusst, dass es diverse Lernzugänge, unzählige Vermittlungs- bzw. Aneignungswege und ebenso viele Lernvoraussetzungen wie Lernergebnisse gibt. Die Bewertung von geeignetem Unterrichtsmaterial gleicht zudem der Beurteilungsaufgabe im Unterricht, die Lehrerinnen und Lehrer kompetent, gerecht und verantwortungsbewusst ausüben sollen. Hierfür sind hohe pädagogisch-psychologische und diagnostische Kompetenzen erforderlich (Beschluss der KMK vom 5. 10. 2000, 3). Die Berufsgruppe der Lehrkräfte ist es deshalb, die in der Qualitätsdebatte zu OER einen professionellen Beitrag leisten kann, um den aktuellen Unsicherheiten und Unbestimmtheiten entgegenzuwirken.

Dafür sollten die Unterstützungsangebote im institutionellen Rahmen ausgebaut werden und ein Handlungsfeld für die Hilfe zur Selbsthilfe geschaffen werden, in welchem Lehrkräfte die Verantwortung übernehmen. Denn die Lehrkräfte sind es auch, die über ihre Unterrichtspraxis den direkten Kontakt zu weiteren Akteursgruppen im Aushandlungsprozess von Qualität haben. Diese bilden durch jeweils unterschiedliche Ansprüche ein sich stets wandelndes Konstrukt von Qualität. Räume zu bilden, die diese Perspektiven und Ansprüche in den Qualitätsdiskurs einbeziehen, die den Diskurs offen für Modifikationen und Entwicklung halten, haben nicht nur einen Vorsprung gegenüber normativen Qualitätskriterien von Bildungsinstitutionen und Verlagen, sondern können im Merkmal ihrer Offenheit einen Beitrag zu einer Kultur der Teilhabe und des Teilens werden. Vor dem Hintergrund, dass «es keine Instanz gibt, keine Sache und keine Institution, die den autonom urteilenden und wertenden Subjekten unterrichtlichen Handelns die Entscheidung über die Bestimmung und Konkretisierung jener Kriterien abnehmen kann» (Heid 2000, 45), braucht es für die Qualitätssicherung einen kollektiven Aushandlungsprozess, welcher in der 
fortwährenden Nutzung von OER selbst liegt. Dabei darf nicht vernachlässigt werden, dass erst die Präzisierungen und Operationalisierungen individueller Qualitätskriterien und -überzeugungen einen Verständigungsdiskurs ermöglichen.

\section{Fazit: Alternative Perspektive auf Qualität von OER}

Die Debatte um die Qualität von OER ist mit einem notwendigen Wechsel der Betrachtung von Qualität verbunden, der den Steuerungsanspruch der Qualitätssicherung von OER in Frage stellen kann und muss. Es bedarf für OER eines erweiterten Qualitätsverständnisses, das der spezifischen Eigenart von freien Bildungsmaterialien gerecht wird und die freie Zugänglichkeit sowie den Distributions- und Veränderungsgedanken in den Blick nimmt.

Nicht mehr die externe Bewertung von Qualität ist dann erklärtes Ziel der Betrachtung, sondern die Beobachtung und Analyse ${ }^{4}$ dieser. Das impliziert ein Loslassen des Qualitätssicherungsanspruches für eine autonome Entwicklung von OER, die auch Qualitätsverluste toleriert. Eine Beobachtung und Analyse von Qualität über die systemische Makro-, Meso- und Mikroebenen oder über die Annäherung mit Hilfe des Lebenszyklus von OER nimmt zudem wieder die Akteure verstärkt in den Blick und übergeht nicht die Unvereinbarkeit von Kontrollanliegen und freier Interaktion in Bildungsprozessen mit OER. ${ }^{5}$ Damit rücken die Lehrkräfte und Schüler/innen als Beteiligte im Aushandlungsprozess um Qualität von OER für die Schule in den Fokus. Gut ausgebildete Lehrkräfte verfügen über professionelle Bewertungskompetenzen, gehören doch die individuelle Bewertung und systemische Evaluation von Lehr- und Lernprozessen (vgl. KMK 2004) zur Ausbildung von Lehrkräften. Dies kann dem steigenden Bewertungsbedarf gegenüber der Lehr-/Lernkompetenzen und den eingesetzten Bildungsmaterialien in Deutschland entgegenkommen. Wenn der Aushandlungsprozess von Qualität demokratisiert wird, kann dieser mehr als bisher den diversen Bedarfen und Forderungen an freies Bildungsmaterial befriedigen und zu einem nachhaltigen Vertrauen in die Kultur des Teilens führen. Das breite Spektrum der Akteure und Beteiligten muss in einem Qualitätskonzept für OER aufgehen und transparent gemacht werden.

Da für OER die Forderung nach Qualitätssicherung besonders laut ist, sollten für diese Materialien insbesondere standardisierte Qualitätssicherungsverfahren der Bildungsorganisationen diskutiert und neu verhandelt werden, wie es die Studie zur Qualität von OER der HOOU andeutet. Da es sich um freies Bildungsmaterial handelt,

4 Eine Analyse schliesst sowohl die Wertung als auch die Interpretation aus. Sie benennt Beobachtetes möglichst objektiv und bietet Benanntes zur Anschlusskommunikation an.

5 Eine Annäherung an ein angemessenes Qualitätskonzept haben Camilleri, Ehlers und Pawlowski über den «lifecycle of a learning resource» versucht. Die Autoren plädieren in ihrem 2014 vorgelegten Report nachvollziehbar vor dem Hintergrund der Open Educational Practice dafür, das jeweilige Akteursfeld zu den unterschiedlichen Prozessschritten im Lebenszyklus einer OER differenziert einzubeziehen. 
das, bis auf eine Ausnahme, durch die (potentielle) Veränderungsmöglichkeit charakterisiert ist, entzieht es sich durch dieses inhärente Merkmal den bewährten Qualitätsmanagementfunktionen, wie Controlling, Legitimation und Marketing. Das Steuerungsanliegen von Qualitätssicherung trifft bei OER auf das Autonomiestreben der Open Education. Dieser Disparität kann nur mit einer konsequent systemischen und konstruktivistischen Betrachtung der Qualität nachhaltig begegnet werden, die die Organisationen der Bildungsanbieter, den Content selbst, als auch die Perspektiven der diversen Akteure in der Vermittlungspraxis in ihren relationalen Wechselwirkungen massgeblich einbezieht.

\section{Literatur}

Camilleri, Anthony F., Ulf Daniel Ehlers, und Jan Pawlowski. 2014. State of the Art Review of Quality Issues related to Open Educational Resources (OER). Luxemburg: Publications Office of the European Union. https://doi.org/10.2791/80171.

Deimann, Markus, und Theo Bastiaens. 2010. «Potenziale und Hemnisse freier digitaler Bildungsressourcen - eine Delphi Studie». Zeitschrift für E-Learning 5 (3): 7-18. https:// www.researchgate.net/profile/Markus_Deimann2/publication/259784331_Potenziale_und_Hemmnisse_freier_digitaler_Bildungsressourcen_-_eine_Delphi_Studie/ links/542127ca0cf241a65a1e64a9.pdf.

Heid, Helmut. 2000. «Qualität. Überlegungen zur Begründung einer pädagogischen Beurteilungskategorie». Zeitschrift für Pädagogik. 41. Beiheft: Qualität und Qualitätssicherung im Bildungsbereich; Schule, Sozialpädagogik, Hochschule, herausgegeben von Andreas Helmke, Walter Hornstein und Ewald Terhart. Weinheim: Beltz: 41-51. http://nbn-resolving.de/urn:nbn:de:0111-opus-84846.

Kerres, Michael. 2016. «Open Educational Resources (OER)». In Enzyklopädie der Wirtschaftsinformatik, Aufl. 9, hrsg. von Norbert Gronau, Jörg Becker, Elmar J. Sinz, Leena Suhl und Marco Leimeister. Berlin: GITO. https://learninglab.uni-due.de/sites/default/files/OER-Kerres-EnzyklopaedieWInf.pdf.

Knispel, Karl L. 2008. Qualitätsmanagement im Bildungswesen. Ansätze, Konzepte und Methoden für Anbieter von E-Learning und Blended Learning-Qualifizierungen. Münster: Waxmann.

Mau, Steffen. 2017. Das metrische Wir. Über die Quantifizierung des Sozialen. Berlin: Suhrkamp.

Muuß-Merholz, Jöran, und Felix Schaumburg. 2014. Open Educational Resources (OER) für Schulen in Deutschland 2014. Whitepaper zu Grundlagen, Akteuren und Entwicklungen. Internet \& Gesellschaft Collaboratory e.V. https://open-educational-resources.de/wp-content/uploads/OER-Whitepaper_OER-in-der-Schule-2014.pdf.

Neumann, Dominik. 2015. Bildungsmedien online. Kostenloses Lehrmaterial aus dem Internet: Marktsichtung und empirische Nutzungsanalyse. Bad Heilbrunn: Klinkhardt. 
Sekretariat der Kultusministerkonferenz. 2000. «Aufgaben von Lehrerinnen und Lehrern heute - Fachleute für das Lernen». Berlin: KMK. https://www.kmk.org/fileadmin/veroeffentlichungen_beschluesse/2000/2000_10_05-Aufgaben-Lehrer.pdf.

Sekretariat der Kultusministerkonferenz. 2004. «Standards für die Lehrerbildung: Bildungswissenschaften». Beriln: KMK. https://www.kmk.org/fileadmin/veroeffentlichungen_ beschluesse/2004/2004_12_16-Standards-Lehrerbildung-Bildungswissenschaften.pdf.

Sekretariat der Kultusministerkonferenz. 2016. «Bildung in der digitalen Welt: Strategie der Kultusministerkonferenz». Berlin: KMK. https://www.kmk.org/fileadmin/Dateien/pdf/ PresseUndAktuelles/2016/Bildung_digitale_Welt_Webversion.pdf.

Stamm, Margrit. 2003. Evaluation und ihre Folgen für die Bildung. Eine unterschätzte pädagogische Herausforderung. Münster: Waxmann.

Unger, Alexander. 2012. "Die Kultur des Teilens im digitalen Zeitalter». In Kulturen und Ethiken des Teilens, hrsg. von Wolfgang Sützl, Felix Stalder, Ronald Maier und Theo Hug, 131-147. Innsbruck: university press.

Wiley, David. 2013. Defining the „Open“ in Open Content and Open Educational Resources. http://www.opencontent.org/definition/.

Zawacki-Richter, Olaf, und Kerstin Mayrberger. Hrsg. 2017. "Qualität von OER. Internationale Bestandsaufnahme von Instrumenten zur Qualitätssicherung von Open Educational Resources (OER) - Schritte zu einem deutschen Modell am Beispiel der Hamburg Open Online University». Sonderband zum Fachmagazin Synergie. Hamburg. https://doi. org/10.25592/978.3.924330.61.3. 